OPEN ACCESS

Edited by:

Anna Sebestyén,

Semmelweis University, Hungary

${ }^{*}$ Correspondence:

Yingying $X_{U}$

xuyingying@cmu.edu.cn

Qiang Zhang

zhangqiang8220@163.com

${ }^{\dagger}$ These authors share first authorship

Received: 20 July 2021 Accepted: 28 September 2021 Published: 20 December 2021

Citation:

Tan S, Fu X, Xu S, Qiu P, Lv Z, Xu Y and Zhang Q (2021) Quantification of Ki67 Change as a Valid Prognostic Indicator of Luminal B Type Breast Cancer After

Neoadjuvant Therapy.

Pathol. Oncol. Res. 27:1609972.

doi: 10.3389/pore.2021.1609972

\section{Quantification of Ki67 Change as a Valid Prognostic Indicator of Luminal B Type Breast Cancer After Neoadjuvant Therapy}

\author{
Shirong $\operatorname{Tan}^{1 \dagger}$, Xin $\mathrm{Fu}^{2 \dagger}$, Shouping $X u^{3}$, Pengfei Qiu ${ }^{4}$, Zhidong $L v^{5}$, Yingying $X u^{1 *}$ and \\ Qiang Zhang ${ }^{2 *}$
}

${ }^{1}$ Department of Breast Surgery, The First Affiliated Hospital of China Medical University, Shenyang, China, ${ }^{2}$ Department of Breast Surgery, Cancer Hospital of China Medical University, Liaoning Cancer Hospital \& Institute, Shenyang, China, ${ }^{3}$ Department of Breast Surgery, Harbin Medical University Cancer Hospital, Harbin, China, ${ }^{4}$ Breast Cancer Center, Shandong Cancer Hospital and Institute, Shandong First Medical University and Shandong Academy of Medical Sciences, Jinan, China, ${ }^{5}$ Breast Center, The Affiliated Hospital of Qingdao University, Qingdao, China

Introduction: Ki67 value and its variation before and after neoadjuvant chemotherapy are commonly tested in relation to breast cancer patient prognosis. This study aims to quantify the extent of changes in Ki67 proliferation pre- and post-neoadjuvant chemotherapy, confirm an optimal cut-off point, and evaluate its potential value for predicting survival outcomes in patients with different molecular subtypes of breast cancer.

Methods: This retrospective real-world study recruited 828 patients at the Department of Breast Surgery of the First Affiliated Hospital of China Medical University and the Cancer Hospital of China Medical University from Jan 2014 to Nov 2020. Patient demographic features and disease pathology characteristics were recorded, and biomarkers were verified through immunohistochemistry. Various statistical methods were used to validate the relationships between different characteristics and survival outcomes irrespective of disease-free and overall survival.

Results: Among 828 patients, statistically significant effects between pathological complete response and survival outcome were found in both HER2-enriched and triple-negative breast cancer $(p<0.05)$ but not in Luminal breast cancer $(p>0.05)$. Evident decrease of Ki67 was confirmed after neoadjuvant chemotherapy. To quantify the extent of Ki67 changes between pre- and post-NAC timepoints, we adopted a computational equation termed $\Delta K i 67 \%$ for research. We found the optimal cut-off value to be " $\Delta K i 67 \%=-63 \%$ " via the operating characteristic curve, defining $\Delta K i 67 \%$ $\leq-63 \%$ as positive status and $\Delta K i 67 \%>-63 \%$ as negative status. Patients with positive $\Delta K i 67 \%$ status were $37.1 \%$ of the entire cohort. Additionally, 4.7, 39.9, 34.5 and $39.6 \%$ of patients with Luminal A, Luminal B, HER2-enriched and triple negative breast cancer were

\footnotetext{
Abbreviations: AUC, area under the curve; BMI, body mass index; CI, confidence intervals; DFS, disease-free survival; ER, estrogen receptor; FISH, fluorescent in situ hybridization; HR, Hazard Ratio; HR, hormone receptors; HER2, human epidermal growth receptor-2; IBC-NST, invasive breast carcinoma of no special type; IRB, institutional review board; IHC, Immunohistochemical; NAC, neoadjuvant chemotherapy; NRI, net reclassification improvement; OS, overall survival; PR, progesterone receptor; pCR, pathological complete response; ROC, operating characteristic curve; TN, triple negative.
} 
also validated with positive $\Delta K i 67 \%$ status. The statistically significant differences between $\Delta K i 67 \%$ status and prognostic outcomes were confirmed by univariate and multivariate analysis in Luminal B (univariate and multivariate analysis: $p<0.05$ ) and triple negative breast cancer (univariate and multivariate analysis: $p<0.05$ ). We proved $\Delta K i 67 \%$ as a statistically significant independent prognostic factor irrespective of disease-free or overall survival among patients with Luminal B and triple-negative breast cancer.

Conclusions: $\triangle K i 67 \%$ can aid in predicting patient prognostic outcome, provide a measurement of NAC efficacy, and assist in further clinical decisions, especially for patients with Luminal B breast cancer.

Keywords: breast cancer, prognosis, neoadjuvant chemotherapy, Ki67, tumor response

\section{INTRODUCTION}

Breast cancer is the highest cause of cancer-related morbidity among women worldwide [1]. Established biomarkers, including hormone receptors (HR), estrogen receptor (ER), progesterone receptor (PR), human epidermal growth receptor-2(HER-2), and Ki67 labeling index classify breast cancer into four subtypes: HER2-enriched, triple-negative (TN), and Luminal A and B types [2,3]. Neoadjuvant chemotherapy (NAC) is a standard therapeutic strategy for inoperable breast cancer and for some operable patients who seek decreased primary tumor burden and breast conservation [4]. Patient response to NAC also provides guidance for the long-term systemic therapeutic strategy for each individual patient [5]. A achievement of pathological complete response (pCR), disease-free survival (DFS), and overall survival (OS) [6] were used to estimate treatment efficacy. Only $15-20 \%$ of patients who receive NAC reach pCR [7-9]. Although pCR plays an important role in prognostic prediction and assists in treatment decisions for TN and HER2-enriched breast cancer, it is less effective in Luminal breast cancer subtypes [10-12]. Luminal breast cancer still lacks indicators to classify patients who will benefit from NAC.

Ki67, a nuclear indicator of cellular proliferation, has been extensively studied and scrutinized for several years. Although some studies criticize Ki67 for its lack of reproducibility [13,14], many demonstrate that proliferation index relates to patient outcomes [2,4,15-17]. These study showKi67 expression as useful indicator for breast cancer and a useful prognostic factor for patients with Luminal B and node-positive breast cancer, assisting in clinical decision regarding neoadjuvant endocrine therapy $[18,19]$. Some studies indicates that Ki67 levels pre-NAC can be an independent prognostic predictor for OS and DFS [12,16]. Endocrine therapy can decrease cell proliferation, presenting as changed Ki67 level pre- and postNAC $[20,21]$. In the POETIC clinical phase-3 trial, this change in Ki67 levels was able to guide endocrine therapy decisions for women with ER-positive breast cancer [19]. One commonality across studies was that decreased levels of Ki67 post-NAC compared to pre-NAC holds significant prognostic predictive value $[12,18,22-25]$. However, some researchers contend that post-NAC Ki67 may hold limited prognostic value [26].
Previous researches often define the extent of Ki67 change between pre- and post-NAC simply by subtracting the two values. This definition is simple but insufficient, as illustrated in two scenarios. The first is if pre- and post-NAC Ki67 proliferation are both relatively low, then the extent of the change may not reach the set threshold. In the second, the change may be comparatively large but not large enough to reach the cut-off value. Furthermore, high variation of pre- and post-NAC Ki67 have been classified by several groups, with studies proposing different thresholds of variation based on the attempts.

In this retrospective study, we evaluate the usefulness of Ki67 change before and after NAC for predicting survival outcome across breast cancer molecular subtypes. We further quantify the change in Ki67 by percentage before and after NAC and calculate an optimal threshold to assess its predictive function for long-term survival and its ability to aid in deciding further adjuvant therapy modification across breast cancer subtypes.

\section{METHODS}

\section{Patient Selection Criterion}

This retrospective study included patients with primary breast cancer who were treated with NAC from Jan 2014 to Nov 2020 at the Department of Breast Surgery of the First Affiliated Hospital of China Medical University and the Cancer Hospital of China Medical University.

\section{Patient Inclusion Criteria}

All patients received a minimum of one cycle of NAC ahead of surgery. Patients with cancer in situ were excluded, as were patients with invasive breast cancer before NAC could be incorporated in cohort. Patients who received any kind of treatment prior to NAC or who presented with progressive or metastatic breast cancer were excluded. Patients with previous breast cancer, male patients, and those with synchronous invasion, bilateral, or inflammatory breast cancer were also excluded. 68 cases with incomplete or deficient IHC analysis were also excluded. In total, 828 patients met the above restriction standards and were included. 


\section{Classifications of Patients}

This retrospective study received permission from the institutional review board (IRB) of the First Affiliated Hospital and was in accordance with the Helsinki Declaration. All patients involved in the research gave informed consent in written agreements of specimens used for scientific research. The informed consent of retrospective research involvement could be waived based on the retrospective nature of the study. PreNAC core needle biopsy pathology and post-surgery regular pathology was extracted and saved in a database. Patient characteristics were collected including gender, age at diagnosis, body mass index (BMI), maximum tumor diameter, tumor grade and stage, axillary lymph node status, histologic type, NAC schedule and cycle number, histology grade, and clinical response to NAC. The local extent of breast cancer was measured via breast ultrasound, mammography, breast MRI, chest CT, bone scan and/or hepatobiliary and splenic ultrasound to verify distant metastasis. The final size of local breast cancer in our database was adopted following priorities: breast MRI > breast ultrasound > mammography. Every patient with suspicious lymph-node metastasis suggested by imaging examinations underwent ultrasound-guided core biopsy of ultrasound-graphically abnormal nodes for axillary node metastasis confirmation before starting NAC. The final histological assessments were all analyzed using hematoxylin and eosin staining and immunohistochemical (IHC). The histological type of specimens from incorporated patients was distinguished between two subgroups: general invasive breast carcinoma of no special type (IBC-NST) and Others. The latter group contained special subtypes such as lobular, mucinous and tubular carcinomas with $\geq 90 \%$ of the tumor as a pure special tumor type and mixed IBC-NST with special subtypes. Lymph nodes with micro-metastasis were considered positive, including the maximum diameter over $0.2 \mathrm{~mm}$ or the number of tumor cells over 200. Isolated tumor cells were considered negative.

\section{Assessment of Clinical Effectiveness of Chemotherapy}

Pathological complete response (pCR) was defined as no residual invasive tumor upon hematoxylin and eosin evaluation of the complete resected breast specimen and all sampled lymph nodes (noninvasive breast residuals) (ypT0/is, ypN0).

\section{Immunohistochemistry for Biomarker Detection}

Histopathology is regarded as the gold standard for diagnosis. All breast tumor specimens were acquired from core needle biopsy or surgical resections, and every specimen was affixed into formalinfixed, paraffin-embedded tissue sections for preservation. IHC staining was performed using Dako Autostainer Plus and EnVision Dual Link detection reagent (DAKO; Carpinteria, CA) with DAB (Dako). Biomarker status, including ER, PR and HER2, were defined by IHC in strict accordance with European Quality Assurance guidelines. ER and PR staining were assessed based on the American Society of Clinical

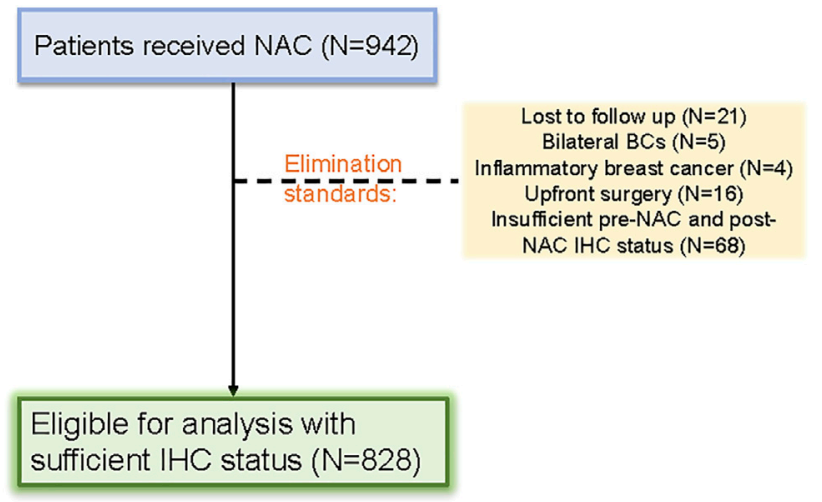

FIGURE 1 | Patient selection flow diagram for the study.

Oncology/College of American Pathologists Guidelines [27,28]. Antibodies used in IHC include as anti-ER (Clone SP1, Dako), anti-PR (Clone PgR636, Dako), and Ki67 (Clone Mib-1, Dako). Hematoxylin II (Dako As Link 48) was used to counterstain specimens automatically. All tests incorporated external positive and negative controls. ER and PR stains were considered positive if immunostaining was seen in more than $1 \%$ of immunoreactive cells. HER2 status was ascertained via IHC using the Hercep Test ${ }^{\mathrm{TM}}$ kit (code K5204, Dako). HER2 expression was scored as 0, 1+, $2+$, and $3+$ according to ASCO guidelines. A score of 3+ was regarded as HER2+, with 0/1+ defined as HER2-. For cases scoring HER2 2+, a fluorescent in situ hybridization (FISH) test could be conducted. The measurement of Ki67 index was based on the spot with the highest intensity in a high-power field (400x) and 500-2000 cells were counted [29].

Following the St. Gallen guidelines 2013 [2], high expression of PR was set as $\geq 20 \%$ and low expression of PR was defined as $<20 \%$. In accordance with the International Ki67 in Breast Cancer Working Group [30], Ki67 index was classified into two groups: low $(<30 \%)$, and high $(\geq 30 \%)$. To quantify the extent of Ki67 changes between pre- and post-NAC timepoints, we used the following equation: [define post-NAC Ki67 as A, define pre-NAC Ki67 as B, computational formula: $\Delta K i 67 \%=(\mathrm{A}-\mathrm{B}) / \mathrm{B} \times 100 \%$, maintaining sign]. If a patient achieved $\mathrm{pCR}$ after NAC, the postNAC Ki67 index was defined as $0 \%$ and $\Delta K i 67 \%$ was mathematically $-100 \%$. Representative IHC staining images of Ki67 subgroups are shown in Supplementary Figure S2.

\section{Breast Cancer Subtypes Definitions}

We classified breast cancers into four subtypes based on HR status, HER2 status and Ki67 index according to the St. Gallen guidelines as follow [2,31]: Luminal A: (ER and PR positive, HER2 negative, "low" Ki-67, and a "low" recurrence risk based on multi-gene-expression assay results if available), Luminal B ["Luminal B-like (HER2 negative)": ER positive, HER2 negative, and at least one of the following: "high" Ki-67, "negative or low" PR, or "high" recurrence risk based on multi-gene-expression assay if available. "Luminal B-like (HER2 positive)": ER positive, HER2 over-expressed or amplified with any Ki-67, and any PR]. HER2-enriched 
TABLE 1 | Demographic and clinicopathological features of whole cohort $(n=828)$

\begin{tabular}{|c|c|}
\hline Parameter & Number (\%) \\
\hline \multicolumn{2}{|l|}{ Age at diagnosis (year) } \\
\hline$<40$ & $83(10.0)$ \\
\hline$\geq 40$ & $745(90.0)$ \\
\hline \multicolumn{2}{|l|}{ BMI $\left(\mathrm{kg} / \mathrm{m}^{2}\right)$} \\
\hline <18.9 (underweight) & $68(8.2)$ \\
\hline $18.9-24.9$ & 361 (43.6) \\
\hline >24.9 (overweight) & 399 (48.2) \\
\hline \multicolumn{2}{|c|}{ Histological type at diagnosis } \\
\hline IBC-NST & $699(84.4)$ \\
\hline Others & $129(15.6)$ \\
\hline \multicolumn{2}{|c|}{ Clinical nodal status at diagnosis } \\
\hline Positive & $676(81.6)$ \\
\hline Negative & $152(18.4)$ \\
\hline \multicolumn{2}{|l|}{ Chemotherapy cycles } \\
\hline$\leq 2$ & $199(24)$ \\
\hline $3-5$ & $424(51.2)$ \\
\hline$>5$ & $205(24.8)$ \\
\hline \multicolumn{2}{|l|}{ Chemotherapy regimen } \\
\hline Taxane -based & $89(10.7)$ \\
\hline Anthracycline-based & $150(18.1)$ \\
\hline Taxane + anthracycline & $589(71.1)$ \\
\hline \multicolumn{2}{|c|}{ Anti-HER2 therapy in patients with HER2-positive $(n=261)$} \\
\hline Yes & $49(18.8)$ \\
\hline No & $212(81.2)$ \\
\hline \multicolumn{2}{|c|}{ Clinical tumor stage at diagnosis } \\
\hline T1 & $80(9.7)$ \\
\hline $\mathrm{T} 2$ & $556(67.1)$ \\
\hline $\mathrm{T} 3 / \mathrm{T} 4$ & $192(23.2)$ \\
\hline \multicolumn{2}{|l|}{ Post-NAC tumor size } \\
\hline$<2 \mathrm{~cm}$ & $434(52.4)$ \\
\hline $2-5 \mathrm{~cm}$ & $355(42.9)$ \\
\hline$>5 \mathrm{~cm}$ & $39(4.7)$ \\
\hline \multicolumn{2}{|l|}{ Response to NAC } \\
\hline $\mathrm{PR} / \mathrm{CR}$ & $494(59.7)$ \\
\hline $\mathrm{SD} / \mathrm{PD}$ & $334(40.3)$ \\
\hline \multicolumn{2}{|l|}{ Achieved pCR } \\
\hline Yes & $138(16.7)$ \\
\hline No & $690(83.3)$ \\
\hline \multicolumn{2}{|l|}{ ER status $^{a}$} \\
\hline Positive & $526(63.5)$ \\
\hline Negative & $302(36.5)$ \\
\hline \multicolumn{2}{|l|}{ PR positivity score ${ }^{\mathrm{b}}$} \\
\hline$<20 \%$ & $520(62.8)$ \\
\hline$\geq 20 \%$ & 308 (37.2) \\
\hline \multicolumn{2}{|l|}{ HER2 } \\
\hline Positive & 261 (31.5) \\
\hline Negative & $447(54.0)$ \\
\hline Unknown & $120(14.5)$ \\
\hline \multicolumn{2}{|l|}{ Pre-NAC Ki67 } \\
\hline$<30 \%$ & $253(30.6)$ \\
\hline$\geq 30 \%$ & 575 (69.4) \\
\hline \multicolumn{2}{|l|}{ Post-NAC Ki67 } \\
\hline$<30 \%$ & 491 (59.3) \\
\hline$\geq 30 \%$ & $337(40.7)$ \\
\hline \multicolumn{2}{|l|}{ Molecular subtypes ${ }^{c}$} \\
\hline Luminal A & $43(5.2)$ \\
\hline Luminal B & $489(59.1)$ \\
\hline HER2-enriched & $148(17.9)$ \\
\hline TNBC & $148(17.9)$ \\
\hline
\end{tabular}

${ }^{a}$ Positivity score $<1 \%$ including negative status.

${ }^{b}$ Positivity score $<20 \%$ including negative status.

"Luminal A: (ER and PR positive, HER2 negative, "low" Ki-67, and a "low" recurrence risk based on multi-gene-expression assay results if available), Luminal B ("Luminal B-like
(HER2 negative)": ER positive, HER2 negative, and at least one of the following: "high" Ki67, "negative or low" $P R$, or "high" recurrence risk based on multi-gene-expression assay if available. "Luminal B-like (HER2 positive)": ER positive, HER2 over-expressed or amplified with any Ki-67, and any PR). HER2-enriched (HER2 over-expressed or amplified, HR absent) and TN (Negative HR and HER2).

$B M I$, body mass index; NAC, neoadjuvant chemotherapy; IBC-NST, invasive breast carcinoma of no special Type; $p C R$, pathological complete response; ER, estrogen receptor; PR, progesterone receptor; HER2, human epidermal growth factor receptor 2; $T N B C$, triple negative breast cancer.

(HER2 over-expressed or amplified, ER and PR absent) and TN (Negative ER, PR and HER2). The cut-point between "high" and "low" values for Ki67 varies and lacks conclusion. 14\% cut-off value of Ki67 for subtype classification was adopted in St. Gallen guidelines 2013 .

\section{Statistical Data Analysis}

Multiple demographic features were analyzed using the Chi-square test. The survival-related indicators studied were DFS and OS. DFS was calculated from the date of initiation of the first regimen to the date of first event (locoregional relapse, distant relapse, or death) and OS was calculated from the date of surgery to the date of death or last follow-up. The Kaplan-Meier method was used to define the difference ratio, and survival curves were compared using the logrank test [32]. Significance was assigned as $p$ value $<0.05$. Receiver operating characteristic curve (ROC) and area under the curve (AUC) were performed to calculate the optimal cut-off value determined by the Youden index with maximum sensitivity and specificity. Cox proportional hazards regression models were used to estimate relapse and survival risk between subgroups. The multivariate Cox proportional hazards model was implemented for Hazard Ratio (HR) and 95\% confidence intervals (CI) to identify independent prognostic factors. Net reclassification improvement (NRI) was used to verify classification accuracy. All statistical data analysis was performed using SPSS 26.0 (SPSS Inc., Chicago, IL, United States) and R programming language (version 3.5.3; https://www.r-project.org/).

\section{RESULTS}

\section{Basic Demographic Features and Baseline Characteristics}

Patients with primary breast cancer who were treated with NAC were selected based on strict standards. 942 patients were initially included in the cohort, but 114 patients were eliminated for various reasons. A total of 828 female patients with primary breast cancer who received NAC were ultimately included in this retrospective study (Figure 1).

Basic demographic and pathologic features are shown in Table 1. The median age of entire cohort was $51 \pm 9.65$ years old (range: $23-76$ years), of which $10.0 \%$ of patients were under 40 years old at diagnosis. Body mass index was used to distinguish subjects: overweight patients with index greater than or equal to 24.9 accounted for $48.2 \%$, underweight patients with index under 18.9 accounted for 8.2 and $43.6 \%$ of patients had a healthy BMI between 18.9-24.9. Breast cancer pathological subtype was 
TABLE 2 | The univariate relationship between above features with pCR $(n=828)$

Parameter

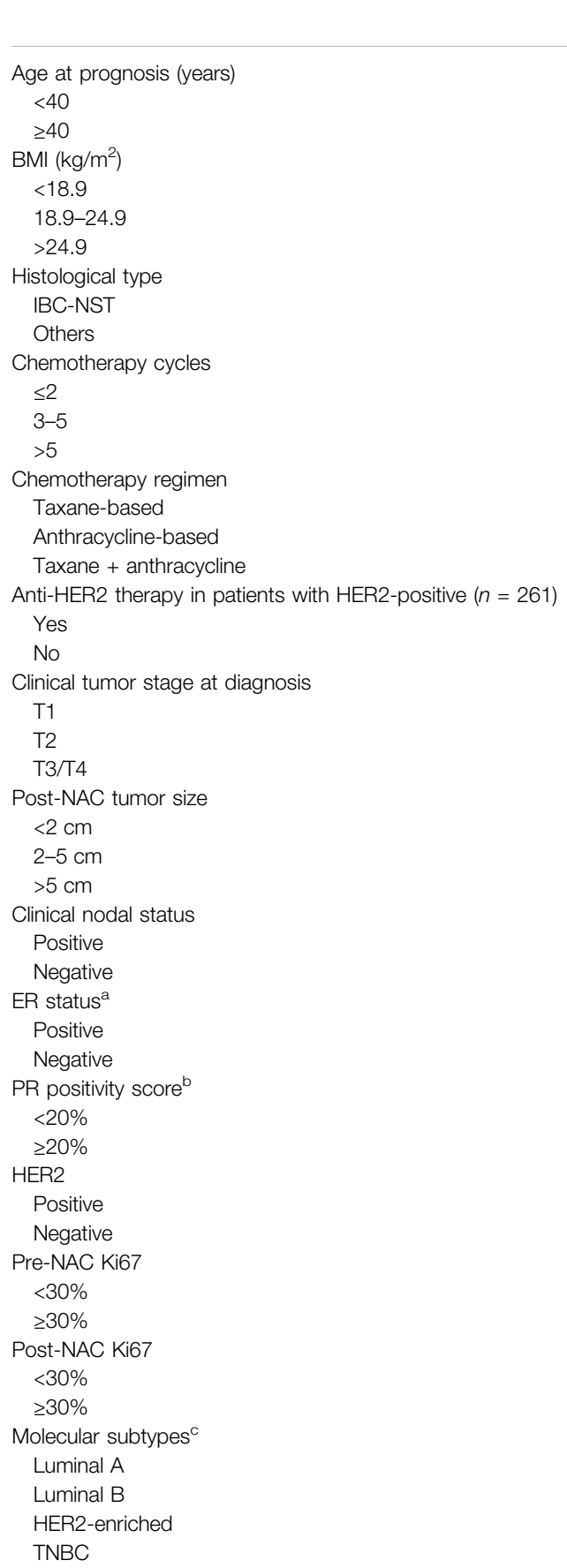

Pathological response to NAC

Non-pCR

p Value

pCR

0.196

18

120

65

625

11

$66 \quad 295$

$61 \quad 338$

$95 \quad 604$

$43 \quad 86$

$30 \quad 169$

$68 \quad 356$

$40 \quad 165$

13

$32 \quad 118$

$93 \quad 496$

$2 \quad 37$

$38 \quad 174$

4466

$85-471$

$39-153$

$04 \quad 330$

$\begin{array}{ll}104 & 330 \\ 30 & 325\end{array}$

4

$99-577$

$39-113$

$75-451$

$63 \quad 239$

$105 \quad 495$

$33-248$

$50 \quad 211$

$73 \quad 374$

$35 \quad 218$

$103 \quad 472$

$119 \quad 372$

$19 \quad 318$

3

$72 \quad 417$

$29-119$

34
0.539

$<0.001$

0.432

0.293

0.267

$<0.001$

$<0.001$

0.014

0.028

0.259

0.147

$<0.001$

0.025

${ }^{a}$ Positivity score $<1 \%$ including negative status.

${ }^{b}$ Positivity score $<20 \%$ including negative status.

'Luminal A: (ER and PR positive, HER2 negative, "low" Ki-67, and a "low" recurrence risk based on multi-gene-expression assay results if available), Luminal B ("Luminal B-like (HER2 negative)": ER positive, HER2 negative, and at least one of the following: "high" Ki-67, "negative or low" PR, or "high" recurrence risk based on multi-gene-expression assay if available. "Luminal B-like (HER2 positive)": ER positive, HER2 over-expressed or amplified with any Ki-67, and any PR). HER2-enriched (HER2 over-expressed or amplified, HR absent) and TN (Negative HR and HER2).

BMI, body mass index; NAC, neoadjuvant chemotherapy; IBC-NST, invasive breast carcinoma of no special Type; pCR, pathological complete response; ER, estrogen receptor; PR, progesterone receptor; HER2, human epidermal growth factor receptor 2; TNBC, triple negative breast cancer. 
TABLE 3 | The univariate relationship between above features with DFS and OS $(n=828)$.

\begin{tabular}{|c|c|c|c|c|c|c|}
\hline \multirow[t]{2}{*}{ Parameter } & \multicolumn{3}{|c|}{ DFS $(n=98)$} & \multicolumn{3}{|c|}{ OS $(n=59)$} \\
\hline & Events-free & Events & $p$ Value & Events-free & Events & $p$ Value \\
\hline Age at prognosis (years) & & & 0.299 & & & 0.181 \\
\hline$<40$ & 76 & 7 & & 80 & 3 & \\
\hline$\geq 40$ & 654 & 91 & & 689 & 56 & \\
\hline BMl $\left(\mathrm{kg} / \mathrm{m}^{2}\right)$ & & & 0.045 & & & 0.026 \\
\hline$<18.9$ & 61 & 7 & & 62 & 6 & \\
\hline $18.9-24.9$ & 323 & 38 & & 344 & 17 & \\
\hline$>24.9$ & 346 & 53 & & 363 & 36 & \\
\hline Histological type & & & 0.849 & & & 0.382 \\
\hline IBC-NST & 609 & 90 & & 644 & 55 & \\
\hline Others & 121 & 8 & & 125 & 4 & \\
\hline Chemotherapy cycles & & & 0.721 & & & 0.567 \\
\hline$\leq 2$ & 174 & 25 & & 184 & 15 & \\
\hline $3-5$ & 377 & 47 & & 397 & 27 & \\
\hline$>5$ & 179 & 26 & & 188 & 17 & \\
\hline Chemotherapy regimen & & & 0.204 & & & 0.364 \\
\hline Taxane-based & 77 & 12 & & 84 & 5 & \\
\hline Anthracycline-based & 138 & 12 & & 142 & 8 & \\
\hline Taxane + anthracycline & 514 & 74 & & 542 & 46 & \\
\hline Anti-HER2 therapy in patients with HER2-positive $(n=261)$ & & & 0.771 & & & 0.712 \\
\hline Yes & 44 & 5 & & 47 & 2 & \\
\hline No & 181 & 31 & & 195 & 17 & \\
\hline Clinical tumor stage at diagnosis & & & 0.889 & & & 0.499 \\
\hline $\mathrm{T} 1$ & 70 & 10 & & 72 & 8 & \\
\hline $\mathrm{T} 2$ & 489 & 67 & & 519 & 37 & \\
\hline T3/T4 & 171 & 21 & & 178 & 14 & \\
\hline Post-NAC tumor size & & & 0.871 & & & 0.778 \\
\hline$<2 \mathrm{~cm}$ & 384 & 50 & & 404 & 30 & \\
\hline $2-5 \mathrm{~cm}$ & 311 & 44 & & 328 & 27 & \\
\hline$>5 \mathrm{~cm}$ & 35 & 4 & & 37 & 2 & \\
\hline Clinical nodal status & & & 0.256 & & & 0.669 \\
\hline Positive & 597 & 78 & & 627 & 48 & \\
\hline Negative & 132 & 20 & & 141 & 11 & \\
\hline ER status ${ }^{a}$ & & & 0.377 & & & 0.293 \\
\hline Positive & 471 & 55 & & 494 & 32 & \\
\hline Negative & 259 & 43 & & 275 & 27 & \\
\hline PR positivity score ${ }^{b}$ & & & 0.246 & & & 0.059 \\
\hline$<20 \%$ & 449 & 71 & & 474 & 46 & \\
\hline$\geq 20 \%$ & 281 & 27 & & 295 & 13 & \\
\hline HER2 & & & 0.275 & & & 0.862 \\
\hline Positive & 225 & 36 & & 242 & 19 & \\
\hline Negative & 402 & 45 & & 417 & 30 & \\
\hline Unknown & 103 & 17 & & 110 & 10 & \\
\hline Pre-NAC Ki67 & & & 0.438 & & & 0.607 \\
\hline$<30 \%$ & 227 & 26 & & 237 & 16 & \\
\hline$\geq 30 \%$ & 503 & 72 & & 532 & 43 & \\
\hline Post-NAC Ki67 & & & 0.008 & & & 0.004 \\
\hline$<30 \%$ & 446 & 45 & & 467 & 24 & \\
\hline$\geq 30 \%$ & 284 & 53 & & 302 & 35 & \\
\hline Molecular subtypes ${ }^{c}$ & & & 0.335 & & & 0.571 \\
\hline Luminal A & 42 & 1 & & 42 & 1 & \\
\hline Luminal B & 432 & 57 & & 456 & 33 & \\
\hline HER2-enriched & 128 & 20 & & 137 & 11 & \\
\hline TNBC & 128 & 20 & & 134 & 14 & \\
\hline
\end{tabular}

${ }^{a}$ Positivity score $<1 \%$ including negative status.

${ }^{b}$ Positivity score $<20 \%$ including negative status.

"Luminal A: (ER and PR positive, HER2 negative, "low" Ki-67, and a "low" recurrence risk based on multi-gene-expression assay results if available), Luminal B ("Luminal B-like (HER2 negative)": ER positive, HER2 negative, and at least one of the following: "high" Ki-67, "negative or low" PR, or "high" recurrence risk based on multi-gene-expression assay if available. "Luminal B-like (HER2 positive)": ER positive, HER2 over-expressed or amplified with any Ki-67, and any PR). HER2-enriched (HER2 over-expressed or amplified, HR absent) and TN (Negative HR and HER2).

BMI, body mass index; NAC, neoadjuvant chemotherapy; IBC-NST, invasive breast carcinoma of no special Type; pCR, pathological complete response; ER, estrogen receptor; PR, progesterone receptor; HER2, human epidermal growth factor receptor 2; TNBC, triple negative breast cancer. 


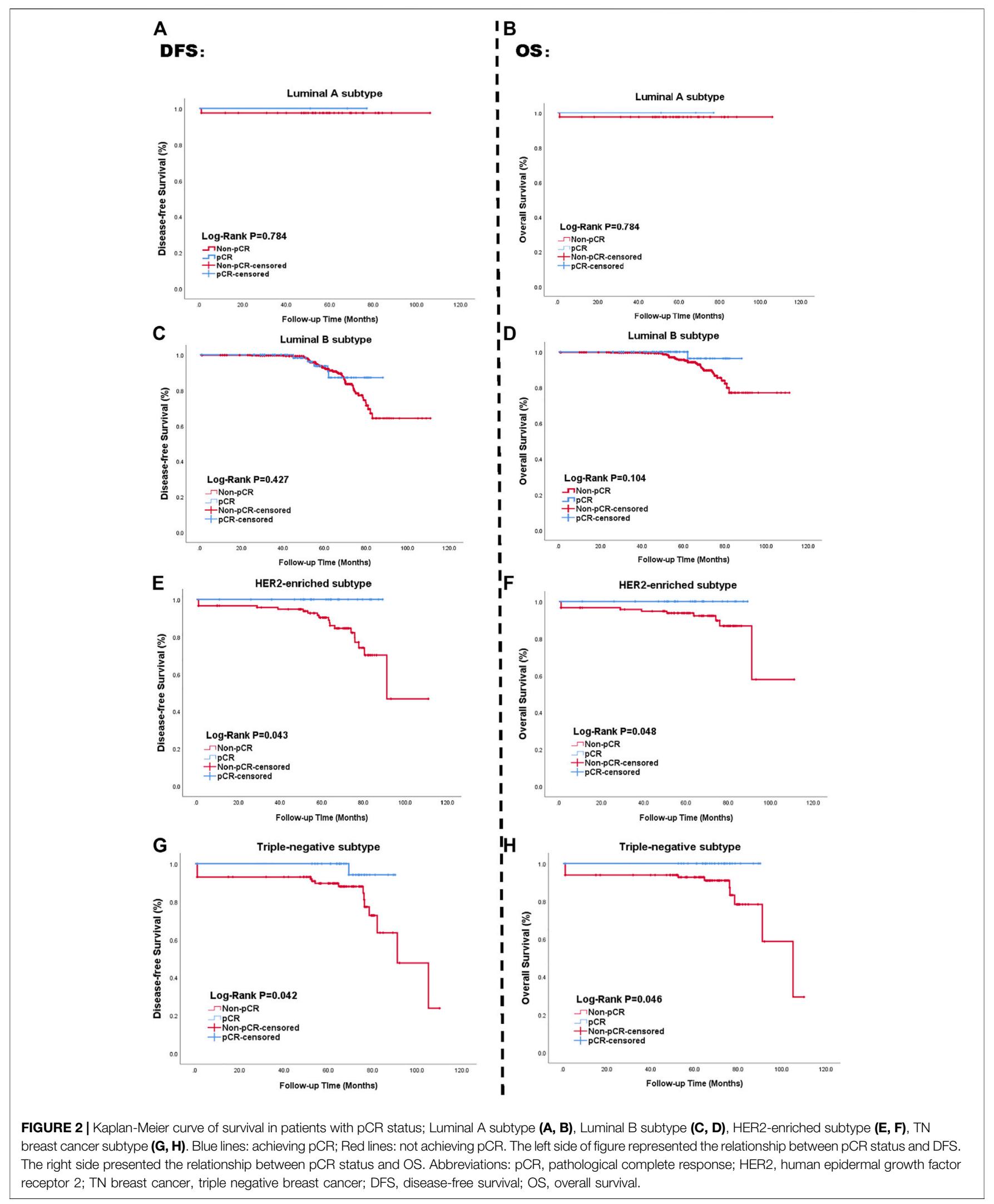



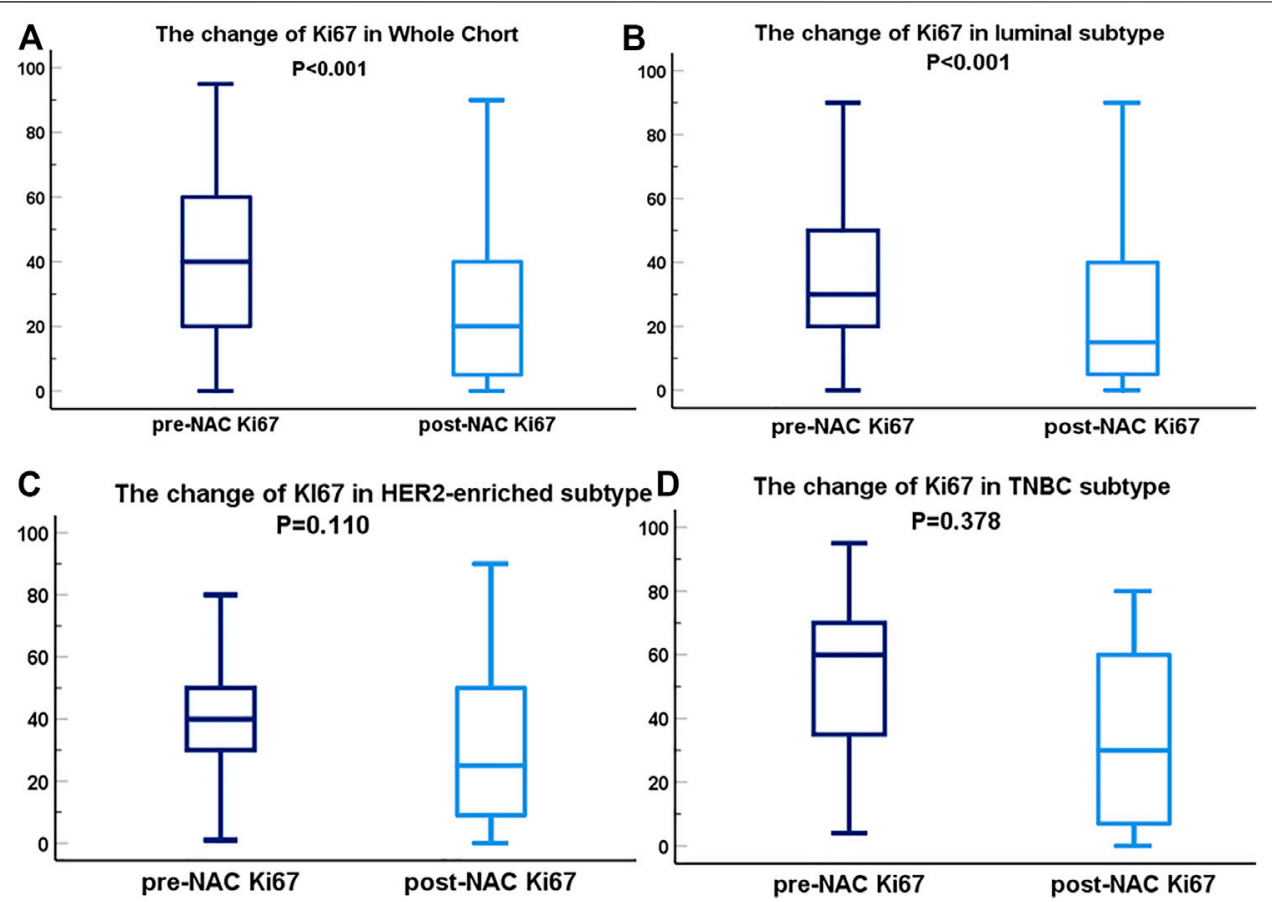

FIGURE 3|Box-plots of pre- and post-NAC Ki67 expression in diverse molecular subtypes; Ki67 variation in whole cohort (A), Luminal subtype (B), HER2 subtype (C) and TN breast cancer subtype (D). The dark blue lines are on behalf of the box-plots of Ki67 before NAC. The light blue lines represent the box-plots of Ki67 after NAC. Abbreviations: pCR, pathological complete response; HER2, human epidermal growth factor receptor 2; TN breast cancer, triple negative breast cancer.

TABLE 4 | The $p$ values of AUCs in different subtype and corresponding $\triangle K i 67 \%$ cut-off point.

\begin{tabular}{lcc} 
Molecular subtype $^{\mathbf{a}}$ & $\boldsymbol{p}$ Value of AUCs & (Ki67\% cut-off point \\
\hline Luminal A & 0.107 & - \\
Luminal B & 0.047 & $-63 \%$ \\
HER2-enriched & 0.131 & - \\
TNBC & 0.009 & $-68 \%$ \\
Whole cohort & 0.004 & $-63 \%$
\end{tabular}

"Luminal A: (ER and PR positive, HER2 negative, "low" Ki-67, and a "low" recurrence risk based on multi-gene-expression assay results if available), Luminal B ("Luminal B-like (HER2 negative)": ER positive, HER2 negative, and at least one of the following: "high" Ki67, "negative or low" $P R$, or "high" recurrence risk based on multi-gene-expression assay if available. "Luminal B-like (HER2 positive)": ER positive, HER2 over-expressed or amplified with any Ki-67, and any PR). HER2-enriched (HER2 over-expressed or amplified, HR absent) and TN (Negative HR and HER2).

$A \cup C$, area under curve.

confirmed as invasive carcinoma of NST for $84.4 \%$ of patients. All other histological types represented $15.6 \%$ of the full cohort. A median of 4 NAC cycles were received (range: 1-9), with NAC classified into three groups: texane-based (10.7\%), anthracyclinebased $(18.1 \%)$ and texane + anthracycline $(71.1 \%)$. The average maximum tumor diameters before and after NAC were $3.41 \pm$ 1.651 and $2.26 \pm 1.648 \mathrm{~cm}$, respectively. $81.9 \%$ patients presented with node-positive status at diagnosis. Finally, 138 subjects (16.7\%) who received NAC achieved pCR, a commonly used measurement of NAC efficacy.

We next analyzed IHC biomarkers. Patients with ER positivity made up $59.5 \%$ of the cohort. Patients with $\mathrm{PR}<20 \%$, or negative status, represented $62.8 \%$ of the cohort. Based on strict IHC staining, subjects positive for HER2 accounted for $31.5 \%$ of cases $87.6 \%$ of all cases had Ki67 expression $\geq 30 \%$ before NAC, while only $58.9 \%$ of the cohort had $\geq 30 \%$ Ki67 after NAC. $18.8 \%$ of HER2-positive patients received anti-HER2 therapy.

Based on biomarker status, patients were categorized into four subtypes: 43 subjects (5.2\%) were categorized as Luminal A subtype breast cancer, 489 subjects (59.1\%) had a Luminal B breast cancer, 148 subjects (17.9\%) had HER2-enriched breast cancer, and 148 cases (17.9\%) had TN breast cancer. IHC status and subtypes distribution are shown in Table $\mathbf{1}$.

\section{Correlation Between Patient Features and Pathological Response to NAC or Survival}

We chose pCR as our evaluation criterion of pathological response to NAC. The median follow-up time was $62.00 \pm$ 21.43 months. A associations between patient features and pathological response to NAC or survival were assessed via the Chi-square test $(\chi 2)$, with results shown in Table 2. Age, body mass index, maximum tumor diameter before NAC, and HER2 status all had $p$ values $>0.05$, indicating no significant influence on prognosis. However, different carcinoma pathology subtypes, maximum diameter after NAC, and nodal status at diagnosis were all significantly associated with pCR, with all $p$ values $<0.05$. The IHC biomarkers ER, PR and Ki67 status before and after NAC, all associated with pathological response to NAC. The log-rank test was used for in analysis of different parameters with DFS and OS fully considering the follow-up time (Table 3). 
TABLE 5 | The univariate analysis of relation between basic characteristics with $\Delta K i 67 \%$ status.

Parameter

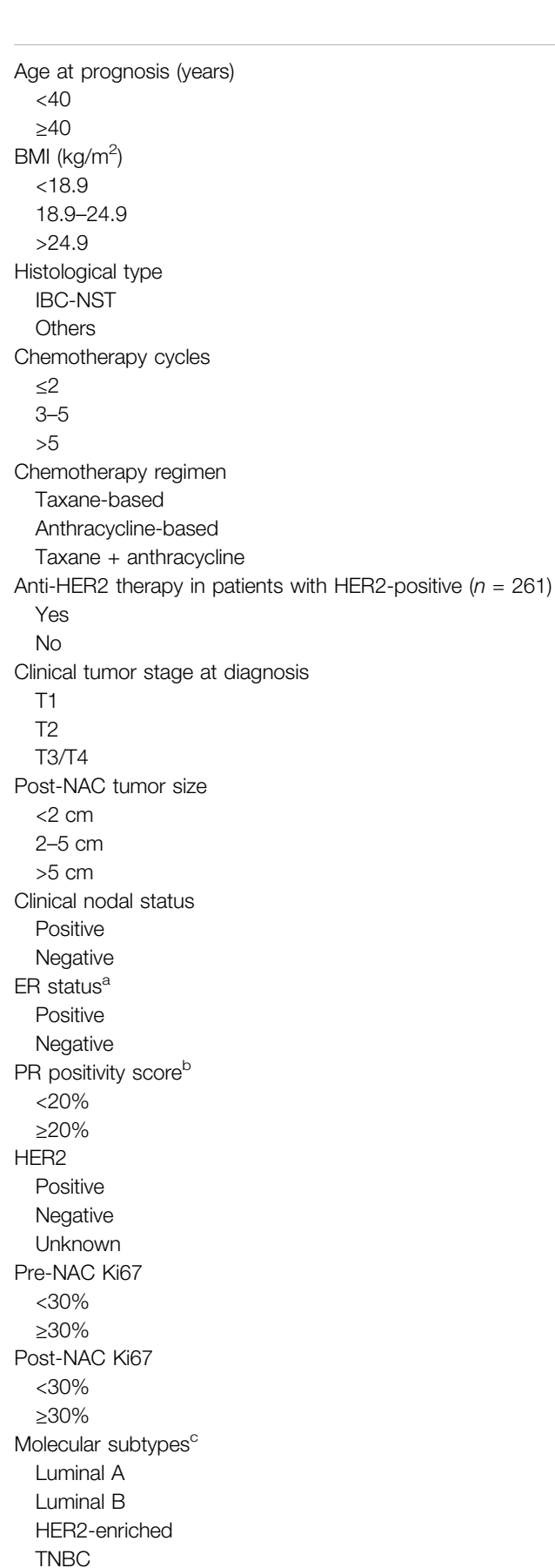

\section{$\Delta K i 67 \%$ status}

$\Delta K i 67 \%>-63 \%$ (Negative)

p Value

$\Delta K i 67 \% \leq-63 \%$ (Positive)

0.105

24

283

59

462

0.208

26

145

136

42

216

263

$228 \quad 471$

$79 \quad 50$

$48-151$

$176 \quad 248$

$83-122$

$26-63$

$81-69$

$200 \quad 389$

$22 \quad 27$

$77 \quad 135$

21

$196 \quad 360$

$90 \quad 102$

$174 \quad 260$

120

$13 \quad 26$

$237 \quad 439$

$70 \quad 82$

$196 \quad 330$

111191

$197 \quad 323$

$110 \quad 198$

$99-162$

$161-286$

$47 \quad 73$

$67 \quad 186$

$240-335$

$303+188$

$4 \quad 333$

$2 \quad 41$

$195 \quad 294$

$51-97$

$59-89$

Positivity score $<1 \%$ including negative status.

${ }^{b}$ Positivity score $<20 \%$ including negative status.

"Luminal A: (ER and PR positive, HER2 negative, "low" Ki-67, and a "low" recurrence risk based on multi-gene-expression assay results if available), Luminal B ("Luminal B-like (HER2 negative)": ER positive, HER2 negative, and at least one of the following: "high" Ki-67, "negative or low" PR, or "high" recurrence risk based on multi-gene-expression assay if available. "Luminal B-like (HER2 positive)": ER positive, HER2 over-expressed or amplified with any Ki-67, and any PR). HER2-enriched (HER2 over-expressed or amplified, HR absent) and TN (Negative HR and HER2).

BMI, body mass index; NAC, neoadjuvant chemotherapy; IBC-NST, invasive breast carcinoma of no special Type; pCR, pathological complete response; ER, estrogen receptor; PR, progesterone receptor; HER2, human epidermal growth factor receptor 2; TNBC, triple negative breast cancer. 


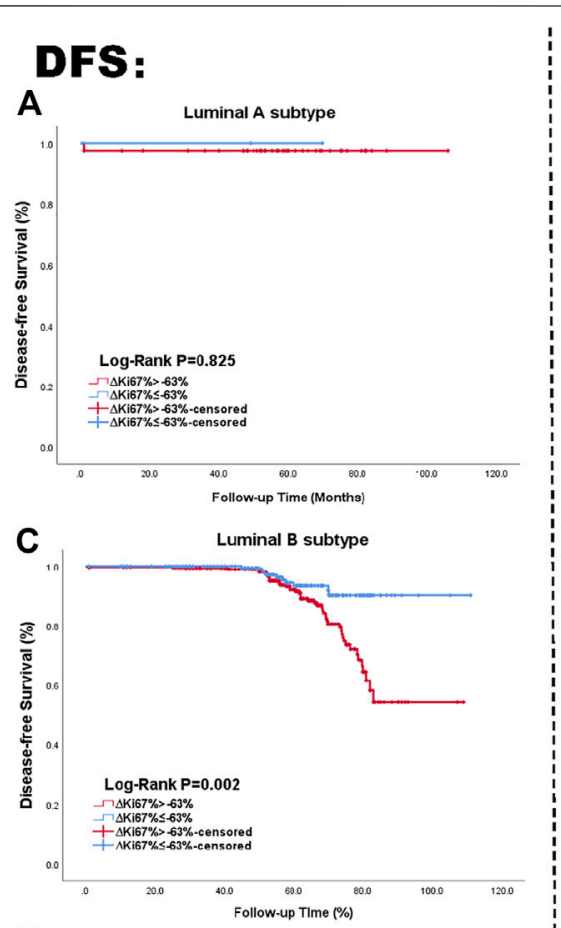

\section{os:}

B
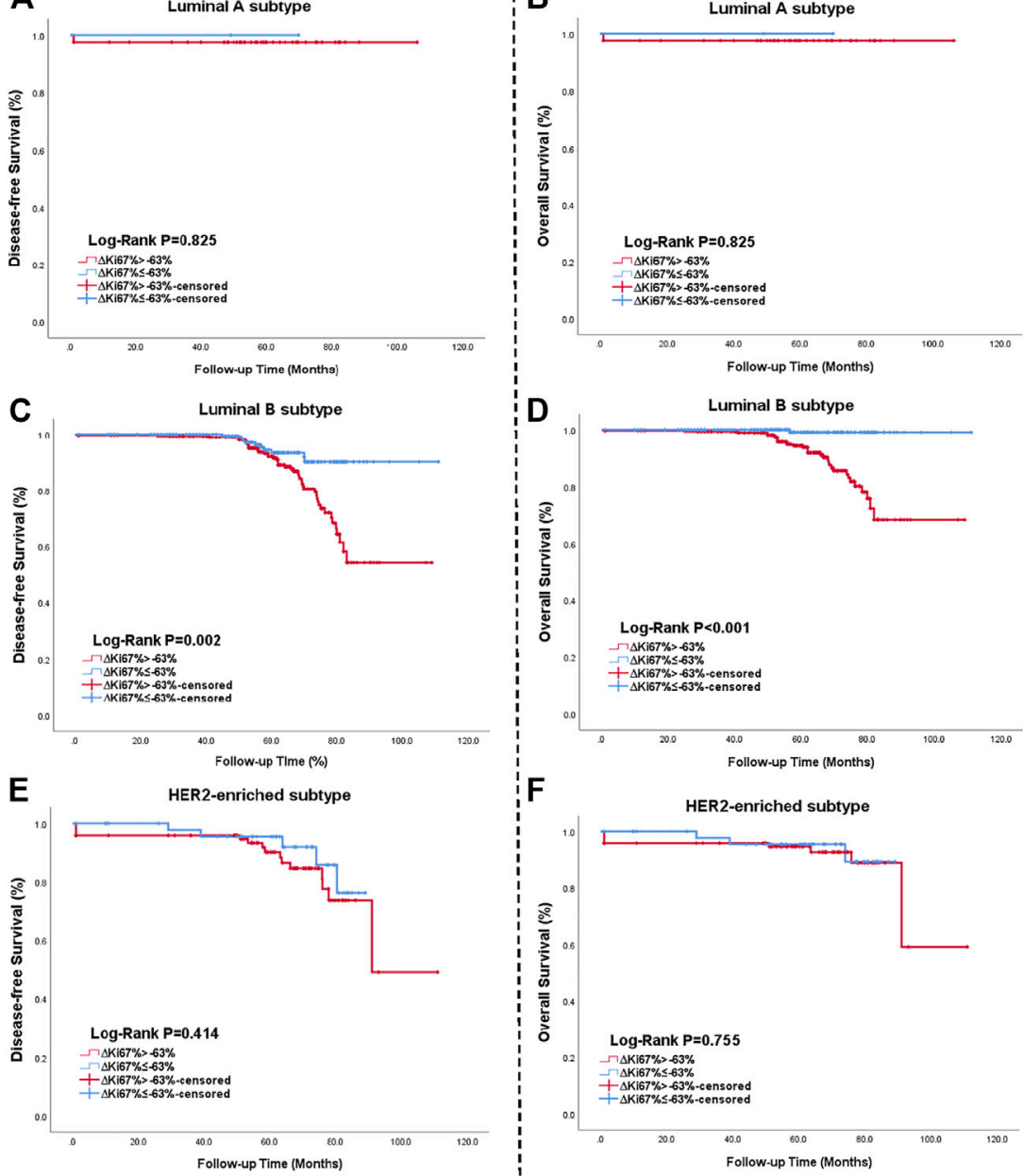

Log-Rank $\mathbf{P}<0.001$

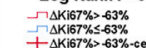

$+\Delta K i 67 \%>-63 \%$-ensored
$+\Delta K 67 \% \% \leq 63 \%$-censored
+
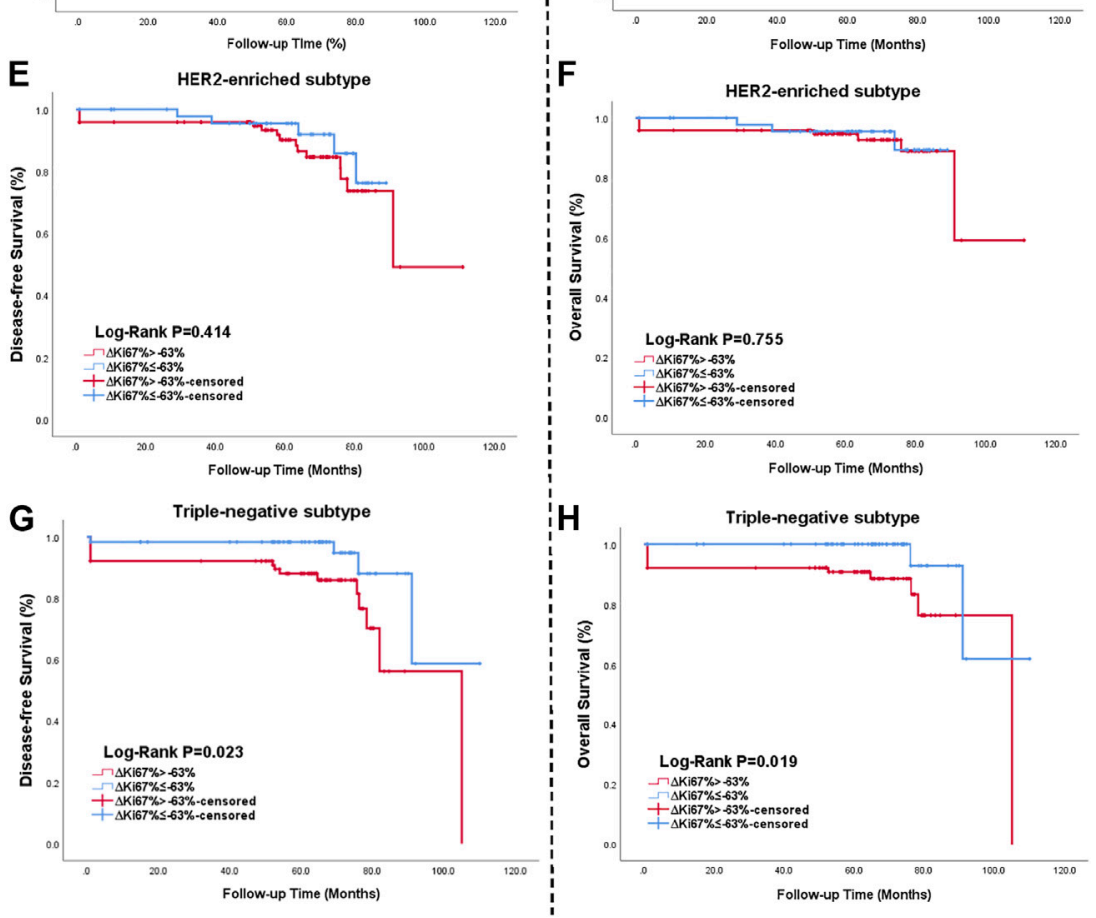

FIGURE 4 | Kaplan Meier curve of DFS and OS in patients with $\triangle K i 67 \%$ status; Luminal A subtype (A, B), Luminal B subtype (C, D), HER2-enriched subtype (E, F), TN breast cancer subtype (G, H). The blue lines are on behalf of achieving $\Delta K i 67 \%$-positive status after NAC. The red lines represented the $\Delta K i 67 \%$-negative status after NAC. The left side of the figure shows the relationship between $\Delta K i 67 \%$ and DFS. The right side of the figure shows the relationship between $\Delta K i 67 \%$ and OS. Abbreviations: HER2, human epidermal growth factor receptor 2; TN, triple negative breast cancer; DFS, disease-free survival; OS, overall survival.

For DFS and OS, BMI and Ki67 after NAC both presented $p$ value $<0.05$.

Histological subtype further affected both pCR rates and survival status. Each subtype resulted in different pathological responses, as validated by the Chi-square test $(p<0.05)$, but there was no obvious change on patient prognosis $(p=0.244>$
0.05). Rates of pCR across subtypes are shown in Figure 2. The left side of the picture presents the relation between $\mathrm{pCR}$ and DFS. The right side of the picture presents the univariate analysis of the relationship between OS and pCR based on the Kaplan-Meier method. We calculated survival rates for patients with each subtype using the Kaplan-Meier method. 
TABLE 6 | The multivariate Cox analysis of $\Delta K i 67 \%$ status in NAC-treated luminal-B subtype patients.

\begin{tabular}{|c|c|c|c|c|}
\hline \multirow[t]{2}{*}{ Parameter } & \multicolumn{2}{|c|}{ Disease-free survival } & \multicolumn{2}{|c|}{ Overall survival } \\
\hline & HR (95\% Cl) & $p$ Value & HR $(95 \% \mathrm{Cl})$ & $p$ Value \\
\hline Age at diagnosis (year) & & 0.741 & & 0.718 \\
\hline$<40$ & 1.000 & & 1.000 & \\
\hline$\geq 40$ & $1.171(0.460-2.983)$ & & $1.251(0.370-4.232)$ & \\
\hline BMl $\left(\mathrm{kg} / \mathrm{m}^{2}\right)$ & & 0.001 & & 0.172 \\
\hline$<18.9$ (underweight) & 1.000 & & 1.000 & \\
\hline $18.9-24.9$ & $4.425(1.288-15.203)$ & 0.018 & $1.347(0.292-6.217)$ & 0.702 \\
\hline >24.9 (overweight) & 7.044 (2.015-24.625) & 0.002 & $2.651(0.591-11.899)$ & 0.203 \\
\hline Histological type & & 0.625 & & 0.961 \\
\hline IBC-NST & 1.000 & & 1.000 & \\
\hline Others & $0.732(0.209-2.565)$ & & Not applicable & \\
\hline Clinical nodal status at diagnosis & & $<0.001$ & & 0.022 \\
\hline Positive & 1.000 & & 1.000 & \\
\hline Negative & $0.264(0.137-0.511)$ & & $0.240(0.071-0.817)$ & \\
\hline Chemotherapy cycles & & 0.310 & & 0.114 \\
\hline$\leq 2$ & 1.000 & & 1.000 & \\
\hline $3-5$ & $0.975(0.466-2.041)$ & 0.947 & $1.916(0.616-5.957)$ & 0.261 \\
\hline$>5$ & $1.597(0.736-3.464)$ & 0.236 & 3.297 (1.033-10.529) & 0.044 \\
\hline Chemotherapy regimen & & 0.216 & & 0.474 \\
\hline Taxane-based & 1.000 & & 1.000 & \\
\hline Anthracycline-based & 0.307 (0.081-1.154) & 0.080 & $0.326(0.051-2.076)$ & 0.236 \\
\hline Taxane + anthracycline & $0.538(0.206-1.403)$ & 0.205 & $0.540(0.156-1.874)$ & 0.332 \\
\hline Clinical tumor stage at diagnosis & & 0.585 & & 0.211 \\
\hline $\mathrm{T} 1$ & 1.000 & & 1.000 & \\
\hline T2 & $0.735(0.327-1.651)$ & 0.456 & $0.429(0.167-1.102)$ & 0.079 \\
\hline $\mathrm{T} 3 / \mathrm{T} 4$ & $0.977(0.389-2.456)$ & 0.961 & $0.561(0.185-1.697)$ & 0.306 \\
\hline Post-NAC tumor size & & 0.383 & & 0.796 \\
\hline$<2 \mathrm{~cm}$ & 1.000 & & 1.000 & \\
\hline $2-5 \mathrm{~cm}$ & $1.473(0.819-2.651)$ & 0.196 & $1.306(0.597-2.857)$ & 0.504 \\
\hline$>5 \mathrm{~cm}$ & $1.760(0.481-6.441)$ & 0.393 & $1.056(0.125-8.896)$ & 0.960 \\
\hline Post-NAC Ki67 & & 0.454 & & 0.525 \\
\hline$<30 \%$ & 1.000 & & 1.000 & \\
\hline$\geq 30 \%$ & $0.793(0.432-1.456)$ & & $0.786(0.375-1.649)$ & 0.635 \\
\hline$\Delta K i 67 \%$ & & 0.001 & & 0.003 \\
\hline$\leq-63 \%$ & 1.000 & & 1.000 & \\
\hline$>-63 \%$ & 3.495 (1.723-7.088) & & 23.024 (2.956-179.333) & \\
\hline
\end{tabular}

BMI, body mass index; NAC, neoadjuvant chemotherapy; IBC-NST, invasive breast carcinoma of no special type.

pCR status had no impact on DFS outcome for patients with Luminal A $(p=0.784>0.05$, Figure 2A) or Luminal B subtypes $(p=0.427>0.05$, Figure 2B). However, both HER2-enriched and TN breast cancer subtype patients showed a significant association between pathological response to NAC and survival outcome $(p=0.043<0.05$, and $p=0.042<0.05$, respectively, Figures 2C,D). The corresponding Kaplan-Meier curves of pCR with OS outcome for four subtypes are presented in Figures $2 \mathrm{E}-\mathbf{H}$. The multivariate Cox analysis for the full cohort is available in Supplementary Table S1. The visual result of univariate analysis displayed via Kaplan-Meier curves is in Supplementary Figure S1.

\section{Assessment of the Prognostic Efficacy of Ki67 Expression Status Before and After NAC}

The average Ki67 status before NAC was $39.66 \% \pm 22.61 \%$. In comparison, the average value after NAC was $25.00 \% \pm 22.91 \%$. The downward trend of Ki67 before and after NAC is displayed in
Figure 3 for the whole cohort (A), Luminal subtype (B), HER2enriched subtype (C), and TN subtype (D). As shown in the figure, Ki67 change before and after NAC presented a statistical difference $(p<0.001)$ in Luminal subtype. However, in HER2enriched and TN breast cancer, the difference of Ki67 represented no statistical significance. To evaluate the degree of Ki67 decline, we compared post-NAC and pre-NAC proliferation indices [define post-NAC Ki67 as A, define pre-NAC Ki67 as B, the computational formula was $\Delta K i 67 \%=(A-B) \div B \times 100 \%$, maintaining sign].

We next used ROC curve analysis to determine the optimal cutoff value for $\Delta K i 67 \%$. Performing the calculations on SPSS 26.0, we found that $\Delta K i 67 \%$ had prognostic efficacy on survival outcomes among the full cohort. Based on the ROC calculation results in all patients, we defined an optimal cut-off of $\Delta K i 67 \% \leq-63 \%(p<$ $0.05)$. The $\Delta K i 67 \%$ cut-off point in the Luminal B subtype was “-63\%" ( $p=0.047)$. Meanwhile the cut-off point in the TN breast cancer subtype was “-68\%” ( $p=0.009)$ (Table 4).

Therefore, we defined a reduction of greater than $63 \%$ $(\Delta K i 67 \% \leq-63 \%)$ as $\Delta K i 67 \%$ positive, and a reduction of less than $63 \%(\Delta \mathrm{Ki} 67>-63 \%)$ as $\Delta K i 67 \%$ negative. Positive $\Delta K i 67 \%$ 
A

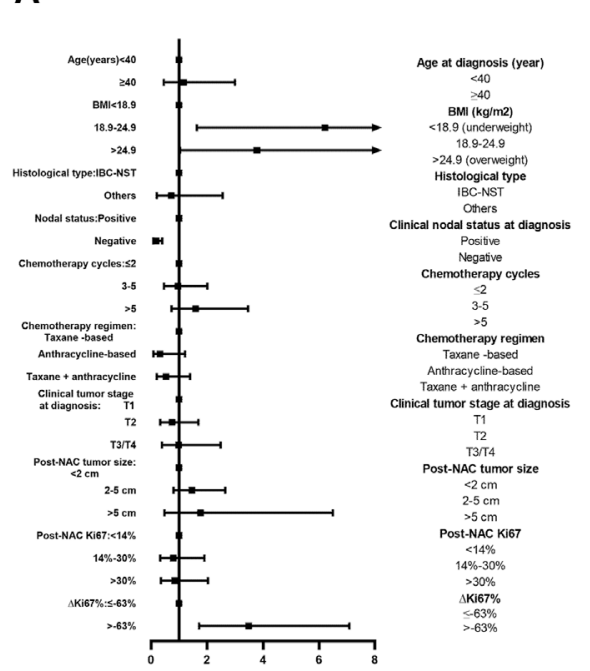

Forest Plot of DFS in luminal-B subtype

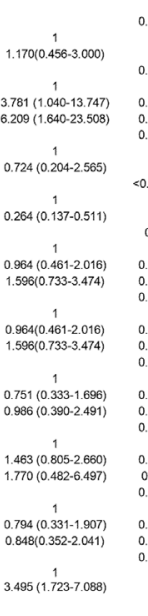

B

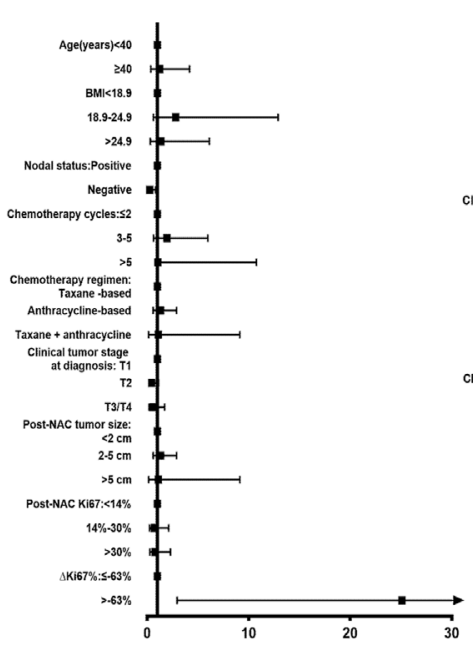

Forest Plot of OS in luminal-B subtype

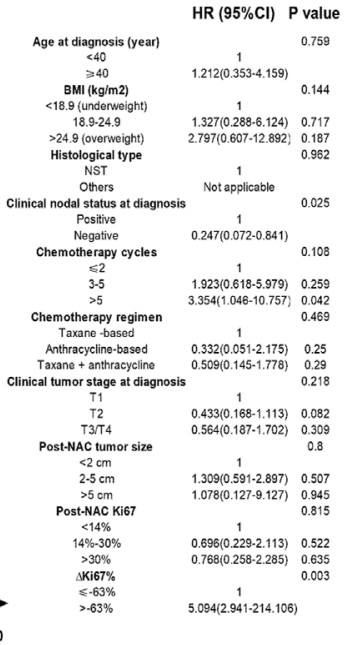

FIGURE 5 | Forest plot of DFS and OS in Luminal B breast cancer; The left side of the figure is exhibiting the forest plot of DFS in Luminal B subtype. The right side of the figure presents the forest plot of OS in Luminal B subtype. The two forest plots both present the multivariate-analyses results; Abbreviations: BMI, body mass index; NAC, neoadjuvant chemotherapy; IBC-NST, invasive breast carcinoma of no special type; DFS, disease-free survival; OS, overall survival.

status presented a larger magnitude of change for $\Delta K i 67 \%$ between pre- and post- NAC, with negative status showing opposite. We used Chi-square tests to evaluate the relationship between demographic and pathological features and $\Delta K i 67 \%$ status (Table 5). $\Delta K i 67 \%$ -positive patients represented $37.1 \%$ of the full cohort. Histological type, number of chemotherapy cycles, type of chemotherapy regimen, clinical nodal status, molecular subtypes, pre- and postNAC tumor size, and Ki67 all showed statistically significant relationship with $\Delta K i 67 \%$ status $(p$ values $<0.05)$.

In summary, $\Delta K i 67 \%$-positive status related with better survival outcomes. We used the Kaplan-Meier method to affirm the correlation between $\Delta K i 67 \%$ status and survival outcomes in each molecular subtype. Survival curves of DFS and OS based on $\Delta K i 67 \%$ status for the four subtypes are displayed in Figures 4A-H. Similar to Figure 2, the left side of the figure presents the Kaplan-Meier curves related with DFS and $\Delta K i 67 \%$, with the right side presenting the relationship between OS and $\Delta K i 67 \%$. As shown in the figure, the univariate log-rank test demonstrated $\Delta K i 67 \%$ was statistically significantly related to DFS and OS in Luminal B and TN subtype, while it showed no definite effect in Luminal A and HER2-enriched subtypes (all $p>0.05$ ).

Based on the multivariate Cox analysis, $\Delta K i 67 \%$ status is a significant independent prognostic predictor of survival outcome regardless of DFS and OS, with DFS-HR $=3.495(95 \% \mathrm{CI}$ 1.723-7.088, $p=0.001)$ and OS-HR $=23.024(95 \% \mathrm{CI}$ 2.956-179.333, $p=0.003$ ) for the Luminal B subtype (Table 6, corresponding forest plot in Figure 5.

Not only that, we tentatively continued to explore the subgroups of Luminal B patients based on the HER2-status. In Luminal B patients from our research, who with negative HER2 status were 256 (52.4\%). The patients with positive HER2 status were 113 (23.3\%). The patients with unknown HER2 status were 120 (24.5\%). The Kaplan-Meier curves and multivariate-analysis results of relationship between $\Delta K i 67 \%$ and survival outcome were attached in the Supplementary Presentation S1. Based on the statistical calculation, the analytical results of all subdivisions in Luminal B tumors fully supported the statistical significance of $\Delta K i 67 \%$ (univariate and multivariate $p<0.05$ ) except the DFS in HER2positive Luminal B subtypes (univariate and multivariate $p>0.05$ ). Combined, $\Delta K i 67 \%$ was confirmed the statistically significant relationship with disease-free and overall survival outcome.

Among patients with TN breast cancer, $\Delta K i 67 \%$ status also provided meaningful survival forecasts on DFS $(p=0.023<0.05)$ and OS $(p=0.019<0.05)$ presented in Figure 4. The relationship between $\Delta K i 67 \%$ status and survival outcomes in the TN breast cancer subtype was confirmed by multivariate Cox analysis as well, with DFS-HR = 3.354 (95\% CI 1.103-10.196, $p=0.033)$ and OS-HR $=30.774(95 \%$ CI 3.552-266.644, $p=0.002)$ (Table 7, corresponding forest plot in Figure 6). Two forest plots represented that negative $\Delta K i 67 \%$ status is a valid indicator for better prognostics. We further used the Kaplan-Meier method to affirm the correlation between $\Delta K i 67 \%$ status and survival outcomes in each molecular subtype. Survival curves based on $\Delta K i 67 \%$ status for the four subtypes are displayed in Figure 6. $\Delta K i 67 \%$ status shows statistically significant differences in Luminal B and TN breast cancer patients. The NRI value comparing the prognostic capacity between $\Delta K i 67 \%$ status and $\mathrm{pCR}$ in TN breast cancer subtype was 0.685 (95\% CI $0.3336-1.0294, p<0.001$ ), also supporting our conclusions. In both Figures 5, $6 \Delta K i 67 \%$ had a statistically significant relationship with prognostic outcome.

\section{DISCUSSION}

NAC is currently widely applied to shrink tumors and decrease carcinoma volume, allowing patients to preserve breasts or 
TABLE 7 | The multivariate Cox analysis of $\triangle K i 67 \%$ status in NAC-treated TNBC subtype patients.

\begin{tabular}{|c|c|c|c|c|}
\hline \multirow[t]{2}{*}{ Parameter } & \multicolumn{2}{|c|}{ Disease-free survival } & \multicolumn{2}{|c|}{ Overall survival } \\
\hline & HR $(95 \% \mathrm{Cl})$ & $p$ Value & HR $(95 \% \mathrm{Cl})$ & $p$ Value \\
\hline Age at diagnosis (year) & & 0.715 & & 0.988 \\
\hline$<40$ & 1.000 & & 1.000 & \\
\hline$\geq 40$ & $1.628(0.173-15.306)$ & & Not applicable & \\
\hline BMl $\left(\mathrm{kg} / \mathrm{m}^{2}\right)$ & & 0.743 & & 0.154 \\
\hline <18.9 (underweight) & 1.000 & & 1.000 & \\
\hline $18.9-24.9$ & $0.462(0.056-3.807)$ & 0.473 & $0.076(0.005-1.056)$ & 0.055 \\
\hline >24.9 (overweight) & $0.444(0.055-3.577)$ & 0.446 & $0.179(0.018-1.834)$ & 0.179 \\
\hline Histological type & & 0.325 & & 0.905 \\
\hline IBC-NST & 1.000 & & 1.000 & \\
\hline Others & $0.429(0.080-2.315)$ & & $1.154(0.109-12.233)$ & \\
\hline Clinical nodal status at diagnosis & & 0.105 & & 0.027 \\
\hline Positive & 1.000 & & 1.000 & \\
\hline Negative & $0.307(0.074-1.281)$ & & $0.132(0.022-0.798)$ & \\
\hline Chemotherapy cycles & & 0.225 & & 0.469 \\
\hline$\leq 2$ & 1.000 & & 1.000 & \\
\hline $3-5$ & $1.647(0.476-5.693)$ & 0.431 & $0.879(0.203-3.811)$ & 0.863 \\
\hline$>5$ & $0.484(0.096-2.449)$ & 0.380 & $0.315(0.043-2.294)$ & 0.254 \\
\hline Clinical tumor stage at diagnosis & & 0.378 & & 0.308 \\
\hline $\mathrm{T} 1$ & 1.000 & & 1.000 & \\
\hline $\mathrm{T} 2$ & $2.265(0.328-15.616)$ & 0.407 & $9.973(0.513-194.007)$ & 0.129 \\
\hline $\mathrm{T} 3 / \mathrm{T} 4$ & 0.557 (0.038 8.260) & 0.671 & $6.405(0.167-246.220)$ & 0.319 \\
\hline Post-NAC tumor size & & 0.462 & & 0.405 \\
\hline$<2 \mathrm{~cm}$ & 1.000 & & 1.000 & \\
\hline $2-5 \mathrm{~cm}$ & $1.048(0.369-2.976)$ & 0.929 & $0.939(0.217-4.060)$ & 0.933 \\
\hline$>5 \mathrm{~cm}$ & $4.088(0.435-38.418)$ & 0.218 & $5.091(0.414-62.551)$ & 0.204 \\
\hline Pre-NAC Ki67 & & 0.137 & & 0.089 \\
\hline$<30 \%$ & 1.000 & & 1.000 & \\
\hline$\geq 30 \%$ & $4.442(0.624-31.635)$ & & $8.686(0.722-104.507)$ & \\
\hline Post-NAC Ki67 & & 0.027 & & 0.032 \\
\hline$<30 \%$ & 1.000 & & 1.000 & \\
\hline$\geq 30 \%$ & $6.880(1.238-38.224)$ & & $7.221(1.181-44.133)$ & \\
\hline$\Delta \mathrm{Ki} 67 \%$ & & 0.033 & & 0.002 \\
\hline$\leq-63 \%$ & 1.000 & & 1.000 & \\
\hline$>-63 \%$ & 3.354 (1.103-10.196) & & 30.774 (3.552-266.644) & \\
\hline
\end{tabular}

BMI, body mass index; NAC, neoadjuvant chemotherapy; IBC-NST, invasive breast carcinoma of no special type.

\section{A Forest Plot of DFS in TNBC subtype}
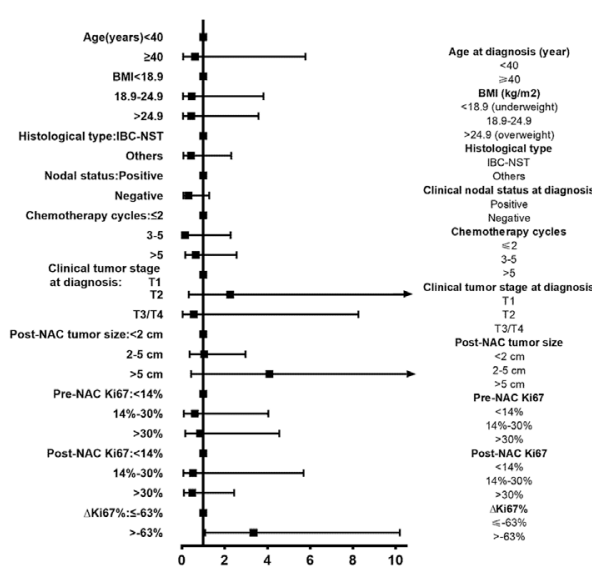
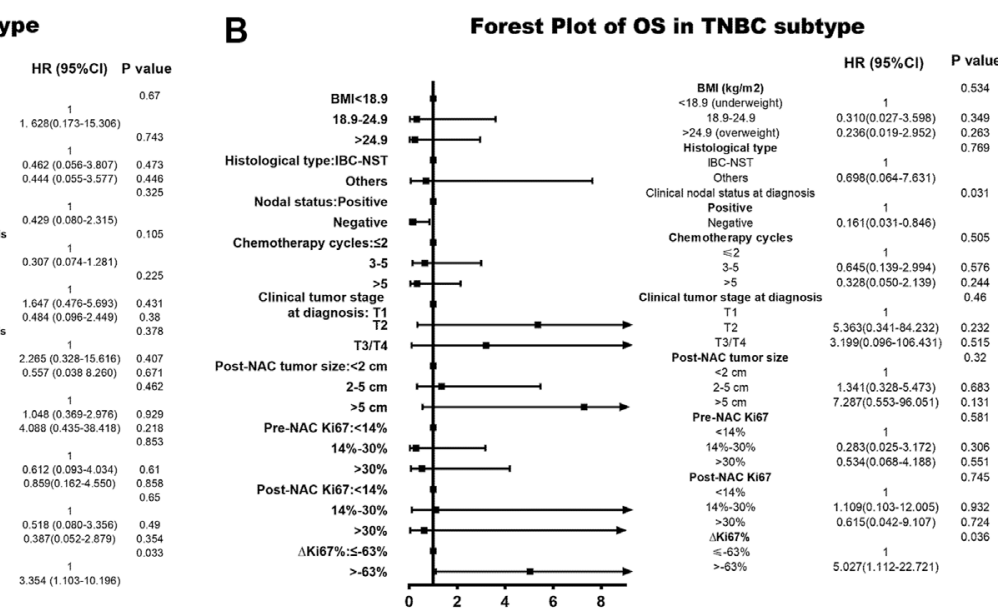

FIGURE 6 | Forest plot of DFS and OS in TN breast cancer. The left side of the figure is exhibiting the forest plot of DFS in triple-negative subtype. The right side of the figure presents the forest plot of OS in triple-negative breast cancer. The two forest plots both present the multivariate-analyses results. Abbreviations: TN, triple negative; BMI, body mass index; NAC, neoadjuvant chemotherapy; IBC-NST, invasive breast carcinoma of no special type; DFS, disease-free survival; OS, overall survival. 
become operable [15]. Moreover, the pathological response to NAC is also beneficial for optimizing chemotherapy regimens and predicting relapse possibility and survival outcomes. Patients with pCR to NAC show improved rates of relapse and better survival $[33,34]$.

Many studies have verified correlations between the degree of Ki67 reduction and pathological response to NAC $[10,35,36]$, yet controversies exist regarding the relationships between Ki67 index, pathological response, and survival grates. Some studies only find significant Ki67 proliferation index differences when comparing pre-NAC with post-surgery in Luminal subtypes [37,38] while other studies have demonstrated that Ki67 reduction also plays a role in TN breast cancer $[39,40]$. While a separate investigation mentioned $\Delta K i 67 \%$, it only discussed its prognostic role and predictive value within 90 Luminal subtype patients with neoadjuvant letrozole-based treatment without classifying it more broadly [20].

In this retrospective real-world study, we analyzed basic demographic and pathological characteristics relative to pCR following to NAC. We confirmed that breast cancer pathological subtype, chemotherapy cycle number, maximum tumor diameter after NAC, nodal state at diagnosis, and Ki67 index pre-NAC and post-NAC all presented statistically significant differences. Furthermore, ER and PR status and molecular subtypes all showed significant effects on pCR rate, as verified in previous studies [41].

As mentioned above, pCR rate ranged from 15 to $20 \%$ in previous studies [7-9]. Patients who achieved pCR following NAC represented $16.7 \%$ of our cohort, a relatively small portion of the total patients. This implies that pCR status increases the specificity of survival outcome predictions but lowered the sensitivity. Many patients were eliminated in the evaluation system, especially those with the Luminal B subtype who represent the largest portion of all breast cancer patients. This is consistent with previous large trials showing $\mathrm{pCR}$ rates to have limited prognostic value in patients with Luminal B subtype $[11,12] . \Delta \mathrm{Ki} 67 \%$ status could help improve this deficiency.

Our study has many strengths. Our fundamental statistical data of post-NAC Ki67 is in accordance with previous research about the relevance of clinical response to NAC and prognostic value $[12,24,25]$. Subtracting pre- and post-NAC Ki67 is insufficient to account for all situations, therefore we used $\Delta K i 67 \%$ as a rational solution. $\Delta K i 67 \%$ is an indicator capable of considering the extent of Ki67 changes in all individuals. Since achievement of pCR is not a useful prognostic indicator in the Luminal B subtype, the field currently lacks efficient parameters to predict outcome and assist in clinical decisions makings for these patients $[11,12]$.

$\Delta \mathrm{Ki} 67 \%$ is a useful indicator for more than just Luminal B subtype patients. In patients with TN breast cancer, pCR rate and $\Delta K i 67 \%$ status both predicted survival outcome with statistical significance. The multivariate analysis confirmed that $\Delta K i 67 \%$ status independently predicted long-term outcomes as well. $\Delta K i 67 \%$ status may be capable to aid with NAC regimen modification with pCR status in the TN subtype. In Luminal B subtypes, we made the research based on different HER2-status subgroups. Nearly all results of subgroups support our conclusions regardless of DFS and OS. Except the HER2-positive Luminal B tumors, the DFS $p$ value of univariate and multivariate analysis is over 0.05 . Meanwhile, considering the function of $\Delta K i 67 \%$ in HER2enriched subtype, it also prompted that HER2(human epidermal growth factor receptor 2) could influence the predictive efficacy of Ki67. The results enlightened us to collect relative data and dig the thoughts deeper.

This study has inherent limitations. Missing data is a common issue in most retrospective single center studies. Hence, we excluded patients whose information was incomplete or inadequate to be incorporated in the study cohort. The second limitation when using Ki67 staining and assessment is lack of stable measurement results [13,14]. To account for this, we adopted the 'hottest-spot' method and performed pathological assessments strictly following international guidelines to improve reproducibility. In the future, artificial intelligence in precision pathology could dramatically improve this method.

In this study, we demonstrate that $\Delta K i 67 \%$ status serves as an independent prognostic factor in Luminal B subtype patients. According to the POETIC clinical phase-3 trial, Ki67 variation in women with operable ER-positive primary breast cancer after preoperative and perioperative aromatase inhibitor (POAI) therapy assisted in deciding further adjuvant endocrine therapy and chemotherapy [19]. This indicates that $\Delta K i 67 \%$ could fill the current gap for predicting prognostic outcomes in Luminal B subtype patients and assist in further clinical treatment decisions to help modify further adjuvant regimens.

\section{CONCLUSION}

In this study, we validated that the extent of Ki67 change before and after NAC, termed $\Delta K i 67 \%$, associates with patient survival outcomes across subtypes. Our statistical calculations defined a cut-off value for $\Delta K i 67 \%$ of $(-63 \%)$. We confirmed that $\Delta K i 67 \%$ status presents an independent prognostic prediction indicator for long-term outcome in Luminal B and TN breast cancer subtypes. As pCR achievement is not a statistically significant predictor for Luminal B subtype patients, $\Delta K i 67 \%$ status may fill this clinical vacancy, assisting with measuring efficacy of neoadjuvant therapy and providing data for adjuvant therapy adjustment [42].

\section{DATA AVAILABILITY STATEMENT}

The raw data supporting the conclusions of this article will be made available by the authors, without undue reservation.

\section{ETHICS STATEMENT}

This study was allowed by the Ethics Committee of the First Affiliated Hospital of China Medical University. The protocol of this study was approved by the institutional review board (IRB) of the First Affiliated Hospital and was in accordance with the Helsinki Declaration (AF-SOP-07-1.1-01/2019-13). Written informed consent for participation was not required for this study in accordance with the national legislation and the institutional requirements. 


\section{AUTHOR CONTRIBUTIONS}

ST analyzed the collected data and wrote the first draft of the manuscript. XF wrote the first draft of the manuscript. XF, SX, PQ, and ZL collected the data and gave assistance on paper revision. YX and QZ were responsible for supervising the whole project and finalizing the manuscript. All authors have read and approved the manuscript.

\section{FUNDING}

This work was supported by the National Natural Science Foundation of China (81773083), Scientific and Technological Innovation Leading Talent Project of Liaoning Province (XLYC1802108), Support Project for Young and Technological Innovation Talents of Shenyang (RC190393) and Chinese Young Breast Experts Research Project (CYBER-2021-A02). These projects provided all the funds necessary for the collection of cases, analyses of results, statistical interpretation of data and for the submission of the manuscript.

\section{CONFLICT OF INTEREST}

The authors declare that the research was conducted in the absence of any commercial or financial relationships that could be construed as a potential conflict of interest.

\section{REFERENCES}

1. Siegel RL, Miller KD, Jemal A, et al. Cancer Statistics, 2020. CA A Cancer J Clin (2020) 70(1):7-30. doi:10.3322/caac.21590

2. Goldhirsch A, Winer EP, Coates AS, Gelber RD, Piccart-Gebhart M, Thürlimann B, et al. Personalizing the Treatment of Women With Early Breast Cancer: Highlights of the St Gallen International Expert Consensus on the Primary Therapy of Early Breast Cancer 2013. Ann Oncol (2013) 24(9): 2206-23. doi:10.1093/annonc/mdt303

3. Cancer Genome Atlas N. Comprehensive Molecular Portraits of Human Breast Tumours. Nature (2012) 490(7418):61-70. doi:10.1038/nature11412

4. Van de Wiel M, Dockx Y, Van den Wyngaert T, Stroobants S, Tjalma WAA, and Huizing MT. Neoadjuvant Systemic Therapy in Breast Cancer: Challenges and Uncertainties. Eur J Obstet Gynecol Reprod Biol (2017) 210:144-56. doi:10.1016/j.ejogrb.2016.12.014

5. von Minckwitz G, Blohmer JU, Costa SD, Denkert C, Eidtmann H, Eiermann W, et al. Response-Guided Neoadjuvant Chemotherapy for Breast Cancer. Jco (2013) 31(29):3623-30. doi:10.1200/JCO.2012.45.0940

6. Cortazar P, Zhang L, Untch M, Mehta K, Costantino JP, Wolmark N, et al. Pathological Complete Response and Long-Term Clinical Benefit in Breast Cancer: the CTNeoBC Pooled Analysis. The Lancet (2014) 384(9938):164-72. doi:10.1016/s0140-6736(13)62422-8

7. Wang-Lopez Q, Chalabi N, Abrial C, Radosevic-Robin N, Durando X, MouretReynier M-A, et al. Can Pathologic Complete Response (pCR) Be Used as a Surrogate Marker of Survival After Neoadjuvant Therapy for Breast Cancer? Crit Rev Oncology/Hematology (2015) 95(1):88-104. doi:10.1016/ j.critrevonc.2015.02.011

8. von Minckwitz G, Fontanella C, et al. Comprehensive Review on the Surrogate Endpoints of Efficacy Proposed or Hypothesized in the Scientific Community Today. JNCI Monogr (2015) 2015(51):29-31. doi:10.1093/jncimonographs/ lgv007

9. Rodrigues-Ferreira S, Nehlig A, Moindjie H, Monchecourt C, Seiler C, Marangoni E, et al. Improving Breast Cancer Sensitivity to Paclitaxel by Increasing Aneuploidy. Proc Natl Acad Sci USA (2019) 116(47):23691-7. doi:10.1073/pnas.1910824116

\section{SUPPLEMENTARY MATERIAL}

The Supplementary Material for this article can be found online at: https://www.por-journal.com/articles/10.3389/pore.2021.1609972/ full\#supplementary-material

Supplementary Figure S1 | The Kaplan-Meier curves of $\Delta K i 67 \%$ in whole cohort. The Supplementary Figure S1 exhibits the results of univariate analysis, KaplanMeier method. The left side of the picture presents the relationship between the $\Delta K i 67 \%$ status and DFS. The right side of the picture shows the relationship between the $\Delta K i 67 \%$ status and OS. The red lines represent the $\Delta K i 67 \%$ -positive status after NAC. The blue lines represent the $\triangle K i 67 \%$-negative status after NAC. Abbreviations: DFS, disease-free survival; OS, overall survival.

Supplementary Figure S2|Representative immunohistochemical staining of Ki67. $\mathrm{Ki} 67<30 \%$ (A, B), $\mathrm{Ki} 67 \geq 30 \%$ (C, D)

Supplementary Presentation S1 | The results of univariate and multivariate analyses in subdivisions of Luminal B patients. The PDF file contains the KaplanMeier curves and tabular-forms of multivariate results regardless of DFS and OS. Luminal B subtype was sorted into four subdivisions: negative HER2 status, positive HER2 status, luminal B subtype excluded HER2-positive status (containing negative and unknown HER2 status) and luminal B subtype excluded HER2-negative status (containing positive and unknown HER2 status). The blue lines are on behalf of achieving $\Delta K i 67 \%$-positive status after NAC. The red lines represented the $\Delta K i 67 \%$ -negative status after NAC. The left side of the figure shows the relationship between $\triangle K i 67 \%$ and DFS. The right side of the figure shows the relationship between $\triangle K i 67 \%$ and OS; Abbreviations: BMI, body mass index; NAC, neoadjuvant chemotherapy; IBC-NST, invasive breast carcinoma of no special type; $\mathrm{pCR}$, pathological complete response; ER, estrogen receptor; PR, progesterone receptor; HER2, human epidermal growth factor receptor 2; TNBC, triple negative breast cancer; DFS, disease-free survival; OS, overall survival.
10. Li L, Han D, Wang X, Wang Q, Tian J, Yao J, et al. Prognostic Values of Ki-67 in Neoadjuvant Setting for Breast Cancer: a Systematic Review and MetaAnalysis. Future Oncol (2017) 13(11):1021-34. doi:10.2217/fon-2016-0428

11. von Minckwitz G, Untch M, Blohmer J-U, Costa SD, Eidtmann H, Fasching PA, et al. Definition and Impact of Pathologic Complete Response on Prognosis After Neoadjuvant Chemotherapy in Various Intrinsic Breast Cancer Subtypes. Jco (2012) 30(15):1796-804. doi:10.1200/JCO.2011.38.8595

12. Fasching PA, Heusinger K, Haeberle L, Niklos M, Hein A, Bayer CM, et al. Ki67, Chemotherapy Response, and Prognosis in Breast Cancer Patients Receiving Neoadjuvant Treatment. BMC Cancer (2011) 11:486. doi:10.1186/1471-2407-11-486

13. Leung SCY, Nielsen TO, Nielsen TO, Zabaglo L, Arun I, Badve SS, et al. Analytical Validation of a Standardized Scoring Protocol for Ki67: Phase 3 of an International Multicenter Collaboration. NPJ Breast Cancer (2016) 2:16014. doi:10.1038/npjbcancer.2016.14

14. Andre F, Arnedos M, Goubar A, Ghouadni A, and Delaloge S. Ki67-no Evidence for its Use in Node-Positive Breast Cancer. Nat Rev Clin Oncol (2015) 12(5):296-301. doi:10.1038/nrclinonc.2015.46

15. Yoshioka T, Hosoda M, Yamamoto M, Taguchi K, Hatanaka KC, Takakuwa E, et al. Prognostic Significance of Pathologic Complete Response and Ki67 Expression after Neoadjuvant Chemotherapy in Breast Cancer. Breast Cancer (2015) 22(2):185-91. doi:10.1007/s12282-013-0474-2

16. Nishimura R, Osako T, Nishiyama Y, Tashima R, Nakano M, Fujisue M, et al. Prognostic Significance of Ki-67 Index Value at the Primary Breast Tumor in Recurrent Breast Cancer. Mol Clin Oncol (2014) 2(6):1062-8. doi:10.3892/ mco. 2014.400

17. Curigliano G, Burstein HJ, Winer EP, Gnant M, Dubsky P, Loibl S, et al. DeEscalating and Escalating Treatments for Early-Stage Breast Cancer: the St. Gallen International Expert Consensus Conference on the Primary Therapy of Early Breast Cancer 2017. Ann Oncol (2017) 28(8):1700-12. doi:10.1093/ annonc/mdx308

18. Criscitiello C, Disalvatore D, De Laurentiis M, Gelao L, Fumagalli L, Locatelli M, et al. High Ki-67 Score Is Indicative of a Greater Benefit From Adjuvant Chemotherapy When Added to Endocrine Therapy in Luminal B HER2 Negative and Node-Positive Breast Cancer. The Breast (2014) 23(1):69-75. doi:10.1016/j.breast.2013.11.007 
19. Smith I, Robertson J, Kilburn L, Wilcox M, Evans A, Holcombe C, et al. Long-Term Outcome and Prognostic Value of Ki67 After Perioperative Endocrine Therapy in Postmenopausal Women With Hormone-Sensitive Early Breast Cancer (POETIC): an Open-Label, Multicentre, Parallel-Group, Randomised, Phase 3 Trial. Lancet Oncol (2020) 21(11):1443-54. doi:10.1016/s1470-2045(20)30458-7

20. Ianza A, Giudici F, Pinello C, Corona S, Strina C, Bernocchi O, et al. $\Delta \mathrm{Ki} 67$ Proliferation Index as Independent Predictive and Prognostic Factor of Outcome in Luminal Breast Cancer: Data from Neoadjuvant Letrozole-Based Treatment. Tumour Biol (2020) 42(6):101042832092530. doi:10.1177/1010428320925301

21. Bazzola L, Foroni C, Andreis D, Zanoni V, Milani M, Cappelletti G, et al. Combination of Letrozole, Metronomic Cyclophosphamide and Sorafenib Is Well-Tolerated and Shows Activity in Patients With Primary Breast Cancer. $\mathrm{Br}$ J Cancer (2015) 112(1):52-60. doi:10.1038/bjc.2014.563

22. Tanei T, Shimomura A, Shimazu K, Nakayama T, Kim SJ, Iwamoto T, et al. Prognostic Significance of Ki67 Index after Neoadjuvant Chemotherapy in Breast Cancer. Eur J Surg Oncol (Ejso) (2011) 37(2):155-61. doi:10.1016/ j.ejso.2010.10.009

23. Avci N, Deligonul A, Tolunay S, Cubukcu E, Fatih Olmez O, Ulas A, et al. Neoadjuvant Chemotherapy-Induced Changes in Immunohistochemical Expression of Estrogen Receptor, Progesterone Receptor, HER2, and Ki-67 in Patients with Breast Cancer. J BUON (2015) 20(1):45-9. https://pubmed. ncbi.nlm.nih.gov/25778295/\#affiliation-1.

24. Kim KI, Lee KH, Kim TR, Chun YS, Lee TH, and Park HK. Ki-67 as a Predictor of Response to Neoadjuvant Chemotherapy in Breast Cancer Patients. J Breast Cancer (2014) 17(1):40-6. doi:10.4048/jbc.2014.17.1.40

25. Chen R, Ye Y, Yang C, Peng Y, Zong B, Qu F, et al. Assessment of the Predictive Role of Pretreatment Ki-67 and Ki-67 Changes in Breast Cancer Patients Receiving Neoadjuvant Chemotherapy According to the Molecular Classification: a Retrospective Study of 1010 Patients. Breast Cancer Res Treat (2018) 170(1):35-43. doi:10.1007/s10549-018-4730-1

26. Guarneri V, Piacentini F, Ficarra G, Frassoldati A, D'Amico R, Giovannelli S, et al. A Prognostic Model Based on Nodal Status and Ki-67 Predicts the Risk of Recurrence and Death in Breast Cancer Patients With Residual Disease After Preoperative Chemotherapy. Ann Oncol (2009) 20(7):1193-8. doi:10.1093/ annonc/mdn761

27. Wolff AC, Hammond MEH, Hicks DG, Dowsett M, McShane LM, Allison KH, et al. Recommendations for Human Epidermal Growth Factor Receptor 2 Testing in Breast Cancer: American Society of Clinical Oncology/College of American Pathologists Clinical Practice Guideline Update. Arch Pathol Lab Med (2014) 138(2):241-56. doi:10.5858/arpa.2013-0953-SA

28. Hammond MEH, Hayes DF, Dowsett M, Allred DC, Hagerty KL, Badve S, et al. American Society of Clinical Oncology/College of American Pathologists Guideline Recommendations for Immunohistochemical Testing of Estrogen and Progesterone Receptors in Breast Cancer. Jco (2010) 28(16):2784-95. doi:10.1200/JCO.2009.25.6529

29. Kobayashi T, Iwaya K, Moriya T, Yamasaki T, Tsuda H, Yamamoto J, et al. A Simple Immunohistochemical Panel Comprising 2 Conventional Markers, Ki67 and P53, Is a Powerful Tool for Predicting Patient Outcome in Luminal-Type Breast Cancer. BMC Clin Pathol (2013) 13:5. doi:10.1186/1472-6890-13-5

30. Nielsen TO, Leung SCY, Rimm DL, Dodson A, Acs B, Badve S, et al. Assessment of Ki67 in Breast Cancer: Updated Recommendations From the International Ki67 in Breast Cancer Working Group. J Natl Cancer Inst (2021) 113(7):808-19. doi:10.1093/jnci/djaa201

31. Goldhirsch A, Wood WC, Coates AS, Gelber RD, Thürlimann B, Senn H-J, et al. Strategies for Subtypes-Dealing With the Diversity of Breast Cancer:
Highlights of the St Gallen International Expert Consensus on the Primary Therapy of Early Breast Cancer 2011. Ann Oncol (2011) 22(8):1736-47. doi:10.1093/annonc/mdr304

32. Schemper M, and Smith TL. A Note on Quantifying Follow-Up in Studies of Failure Time. Controlled Clin Trials (1996) 17(4):343-6. doi:10.1016/01972456(96)00075-x

33. Schott AF, and Hayes DF. Defining the Benefits of Neoadjuvant Chemotherapy for Breast Cancer. Jco (2012) 30(15):1747-9. doi:10.1200/ jco.2011.41.3161

34. Ellis MJ, Suman VJ, Hoog J, Goncalves R, Sanati S, Creighton CJ, et al. Ki67 Proliferation Index as a Tool for Chemotherapy Decisions During and After Neoadjuvant Aromatase Inhibitor Treatment of Breast Cancer: Results from the American College of Surgeons Oncology Group Z1031 Trial (Alliance). Jco (2017) 35(10):1061-9. doi:10.1200/JCO.2016.69.4406

35. Tao M, Chen S, Zhang X, and Zhou Q. Ki-67 Labeling index Is a Predictive Marker for a Pathological Complete Response to Neoadjuvant Chemotherapy in Breast Cancer. Medicine (Baltimore) (2017) 96(51):e9384. doi:10.1097/ MD.0000000000009384

36. Cabrera-Galeana P, Muñoz-Montaño W, Lara-Medina F, Alvarado-Miranda A, Pérez-Sánchez V, Villarreal-Garza C, et al. Ki67 Changes Identify Worse Outcomes in Residual Breast Cancer Tumors after Neoadjuvant Chemotherapy. Oncol (2018) 23(6):670-8. doi:10.1634/theoncologist.20170396

37. Ács B, Zámbó V, Vízkeleti L, Szász AM, Madaras L, Szentmártoni G, et al. Ki67 as a Controversial Predictive and Prognostic Marker in Breast Cancer Patients Treated With Neoadjuvant Chemotherapy. Diagn Pathol (2017) 12(1):20. doi:10.1186/s13000-017-0608-5

38. Kim HS, Park S, Koo JS, Kim S, Kim JY, Nam S, et al. Risk Factors Associated With Discordant Ki-67 Levels Between Preoperative Biopsy and Postoperative Surgical Specimens in Breast Cancers. PLoS One (2016) 11(3):e0151054. doi:10.1371/journal.pone.0151054

39. Keam B, Im S-A, Lee K-H, Han S-W, Oh D-Y, Kim JH, et al. Ki-67 Can Be Used for Further Classification of Triple Negative Breast Cancer Into Two Subtypes With Different Response and Prognosis. Breast Cancer Res (2011) 13(2):R22. doi:10.1186/bcr2834

40. Munzone E, Botteri E, Sciandivasci A, Curigliano G, Nolè F, Mastropasqua M, et al. Prognostic Value of Ki-67 Labeling Index in Patients With NodeNegative, Triple-Negative Breast Cancer. Breast Cancer Res Treat (2012) 134(1):277-82. doi:10.1007/s10549-012-2040-6

41. Battisti NML, True V, Chaabouni N, Chopra N, Lee K, Shepherd S, et al. Pathological Complete Response to Neoadjuvant Systemic Therapy in 789 Early and Locally Advanced Breast Cancer Patients: The Royal Marsden Experience. Breast Cancer Res Treat (2020) 179(1):101-11. doi:10.1007/ s10549-019-05444-0

42. Tyanova S, Albrechtsen R, Kronqvist P, Cox J, Mann M, Geiger T, et al. Proteomic Maps of Breast Cancer Subtypes. Nat Commun (2016) 7:10259. doi:10.1038/ncomms10259

Copyright $\odot 2021 \mathrm{Tan}, \mathrm{Fu}, \mathrm{Xu}$, Qiu, Lv, Xu and Zhang. This is an open-access article distributed under the terms of the Creative Commons Attribution License (CC BY). The use, distribution or reproduction in other forums is permitted, provided the original author(s) and the copyright owner(s) are credited and that the original publication in this journal is cited, in accordance with accepted academic practice. No use, distribution or reproduction is permitted which does not comply with these terms. 